\title{
A Numerical Technique for Solving the Maxwell Model for Free Surface Flows
}

D.M. CARVAlHO ${ }^{1}$, M.F. TOMÉ, J.A. CUMinato, A. CASTElO, V.G. FERREIRA ${ }^{2}$, Instituto de Ciências Matemáticas e de Computação, ICMC, USP, 13566-590 São Carlos, SP, Brasil.

\begin{abstract}
This work is concerned with the development of a numerical technique for solving free surface flows of a Maxwell fluid. The governing equations for the flow of a Maxwell type fluid together with appropriate boundary conditions are given. The free surface stress conditions are treated in details. A novel formulation for calculating the extra stress components on rigid boundaries is given. The numerical technique presented in this work employs the finite difference method on a staggered grid and employs the ideas of the MAC (Marker-and-Cell) method. Numerical results demonstrating that this numerical technique can solve viscoelastic flows governed by the Maxwell model are presented. Moreover, validation results are presented.
\end{abstract}

\section{Introduction}

Non-Newtonian fluid flows with free surfaces appear in many industrial processes: injection moulding (plastic industries), container filling (food industry), ink jet devices, wire coating, among others, are all examples of non-Newtonian free surface flows problems. Many numerical techniques have been proposed over the past three decades to treat non-Newtonian flows. Today there is an intense activity in this area: a general overview of free surface flows can be found in the book of Griebel et al. [4]. Usually, attention has been given to the upper convected Maxwell model or to the Oldroyd-B constitutive equation. Numerical techniques have included finite element methods (eg. Crochet and Keunings [2]), finite volume methods (eg. Mompean and Deville [5]) and finite difference methods (eg. Yoo and Na [8], Tomé et al. [6]). In this paper two-dimensional viscoelastic flows in the presence of moving free surfaces are considered. A numerical technique for solving flows governed by the upper convected Maxwell constitutive equation is developed. The approach employed is based upon the SMAC (Marker-and-Cell) method of Amsden and Harlow [1]. The method described herein is applied to two-dimensional channel flow and jet buckling. The channel flow is used to validate the numerical method presented in this paper and it is shown that viscoelasticity has a strong influence on the jet buckling phenomenon. This paper is organized as follows: the governing equations

\footnotetext{
${ }^{1}$ Financial support of FAPESP grant no. 01/12538-8

${ }^{2}$ Financial support of FAPESP grant no. 2000/03385-0, CNPq grant no. 523141/94-7
} 
are presented and Section 3 presents the boundary conditions. The essence of the method is given in Section 4 while in Section 5 the basic finite difference equations are presented. Section 6 provides validation results and the numerical simulation of jet buckling.

\section{Basic Equations}

The basic equations governing the viscoelastic incompressible flows of a Maxwell fluid are the equation of motion, the mass conservation equation and the constitutive equation for the upper convected Maxwell model which are given by

$$
\begin{aligned}
\mathbf{T}+\lambda \stackrel{\nabla}{\mathbf{T}} & =2 \mu_{0} \mathbf{D}, \\
\rho\left[\frac{\partial \mathbf{u}}{\partial t}+\nabla \cdot(\mathbf{u u})\right] & =-\nabla p+\nabla \cdot \mathbf{T}+\rho \mathbf{g}, \quad \nabla \cdot \mathbf{u}=0
\end{aligned}
$$

where $p$ is the pressure, $\rho$ is the density, $\mathbf{g}$ is the gravity, $\mathbf{T}$ is the extra-stress tensor, $\mathbf{D}$ rate of deformation tensor, $\mu_{0}$ is coeficient of the solvent viscosity and $\lambda$ is a time constant. The convected derivative $\stackrel{\nabla}{\mathbf{T}}$ and the rate of deformation tensor are defined by

$$
\stackrel{\nabla}{\mathbf{T}}=\frac{\partial \mathbf{T}}{\partial t}+\nabla \cdot(\mathbf{u} \mathbf{T})-(\nabla \mathbf{u})^{T} \cdot \mathbf{T}-\mathbf{T} \cdot(\nabla \mathbf{u}), \quad \mathbf{D}=\frac{1}{2}\left[(\nabla \mathbf{u})+(\nabla \mathbf{u})^{T}\right],
$$

respectively. To solve equations (2.1)-(2.2), we employ the splitting (known as EVSS- Elastic-Viscous Stress-Splitting)

$$
\mathbf{T}=\mathbf{S}+2 \mu_{0} \mathbf{D}
$$

where $\mathbf{S}$ represents the non-Newtonian contribution to the extra stress tensor. Introducing (2.4) into (2.1) and (2.2) we obtain

$$
\begin{aligned}
\mathbf{S}+\lambda \stackrel{\nabla}{\mathbf{S}} & =-2 \lambda \mu_{0} \stackrel{\nabla}{\mathbf{D}} \\
\frac{\partial \mathbf{u}}{\partial t}+\nabla \cdot(\mathbf{u u}) & =-\frac{1}{\rho} \nabla p+\frac{\mu_{0}}{\rho} \nabla^{2} \mathbf{u}+\frac{1}{\rho} \nabla \cdot \mathbf{S}+\mathbf{g}, \quad \nabla \cdot \mathbf{u}=0 .
\end{aligned}
$$

We consider two-dimensional Cartesian flows and let $L$ and $U$ denote reference values for length and velocity. By introducing the nondimensionalization

$$
\mathbf{u}=U \overline{\mathbf{u}}, \quad \mathbf{x}=L \overline{\mathbf{x}}, \quad t=\frac{L}{U} \bar{t}, \quad p=\rho U^{2} \bar{p}, \quad \mathbf{S}=\frac{\mu_{0} U}{L} \overline{\mathbf{S}}, \quad \mathbf{g}=g \overline{\mathbf{g}}
$$

equations (2.5) and (2.6) produce the following nondimensional equations (the bars have been dropped for convenience)

$$
\frac{\partial u}{\partial x}+\frac{\partial v}{\partial y}=0
$$




$$
\begin{gathered}
S^{x x}+W e\left(\frac{\partial S^{x x}}{\partial t}+\operatorname{conv}\left(u S^{x x}\right)+\operatorname{conv}\left(v S^{x x}\right)-2 \frac{\partial u}{\partial x} S^{x x}-2 \frac{\partial u}{\partial y} S^{x y}\right)= \\
-2 W e\left[\frac{\partial}{\partial t}\left(\mathrm{D}^{x x}\right)+\operatorname{conv}\left(u \mathrm{D}^{x x}\right)+\operatorname{conv}\left(v \mathrm{D}^{x x}\right)-2\left(\frac{\partial u}{\partial x}\right)^{2}-\left(\frac{\partial u}{\partial y}\right)^{2}-\frac{\partial u}{\partial y} \frac{\partial v}{\partial x}\right] \\
S^{y y}+W e\left(\frac{\partial S^{y y}}{\partial t}+\operatorname{conv}\left(u S^{y y}\right)+\operatorname{conv}\left(v S^{y y}\right)-2 \frac{\partial v}{\partial x} S^{x y}-2 \frac{\partial v}{\partial y} S^{y y}\right)= \\
-2 W e\left[\frac{\partial}{\partial t}\left(\mathrm{D}^{y y}\right)+\operatorname{conv}\left(u \mathrm{D}^{y y}\right)+\operatorname{conv}\left(v \mathrm{D}^{y y}\right)-\frac{\partial v}{\partial x} \frac{\partial u}{\partial y}-2\left(\frac{\partial v}{\partial y}\right)^{2}-\left(\frac{\partial v}{\partial x}\right)^{2}\right] \\
S^{x y}+W e\left(\frac{\partial S^{x y}}{\partial t}+\operatorname{conv}\left(u S^{x y}\right)+\operatorname{conv}\left(v S^{x y}\right)-\frac{\partial v}{\partial x} S^{x x}-\frac{\partial u}{\partial y} S^{y y}\right)= \\
-W e\left[\frac{\partial}{\partial t}\left(\mathrm{D}^{x y}\right)+\operatorname{conv}\left(u \mathrm{D}^{x y}\right)+\operatorname{conv}\left(v \mathrm{D}^{x y}\right)-2 \frac{\partial v}{\partial x} \frac{\partial u}{\partial x}-2 \frac{\partial u}{\partial y} \frac{\partial v}{\partial y}\right] \\
\frac{\partial u}{\partial t}+\operatorname{conv}\left(u^{2}\right)+\operatorname{conv}(v u)=-\frac{\partial p}{\partial x}+\frac{1}{R e}\left[\frac{\partial^{2} u}{\partial x^{2}}+\frac{\partial^{2} u}{\partial y^{2}}+\frac{\partial S^{x x}}{\partial x}+\frac{\partial S^{x y}}{\partial y}\right]+\frac{1}{F_{r}^{2}} g_{x},(2.1 \\
\frac{\partial v}{\partial t}+\operatorname{conv}(u v)+\operatorname{conv}\left(v^{2}\right)=-\frac{\partial p}{\partial y}+\frac{1}{R e}\left[\frac{\partial^{2} v}{\partial x^{2}}+\frac{\partial^{2} v}{\partial y^{2}}+\frac{\partial S^{x y}}{\partial x}+\frac{\partial S^{y y}}{\partial y}\right]+\frac{1}{F_{r}^{2}} g_{y},
\end{gathered}
$$

where $R e=\rho U\left(L / \mu_{0}\right), W e=\lambda(U / L)$ e $F r=\sqrt{L g} / U$ denote the Reynolds number, the Weissenberg number and the Froude number, respectively. Terms like $\operatorname{conv}(u \bullet)$ and $\operatorname{conv}(v \bullet)$ are related to the convective terms $\frac{\partial(u \bullet)}{\partial x}$ and $\frac{\partial(v \bullet)}{\partial y}$. The components of the rate of deformation tensor are defined by (2.3) and are given by

$$
\mathrm{D}^{x x}=\frac{\partial u}{\partial x}, \quad \mathrm{D}^{x y}=\frac{1}{2}\left(\frac{\partial u}{\partial y}+\frac{\partial v}{\partial x}\right), \quad \mathrm{D}^{y y}=\frac{\partial v}{\partial y} .
$$

\section{Boundary Conditions}

To solve equations (2.7)-(2.12) we need to impose boundary conditions for $\mathbf{u}$. On rigid boundaries the velocity must obey the no-slip condition $\mathbf{u}=\mathbf{0}$ while on inflows it is prescribed by $u_{n}=U_{\text {inf }}$ and $u_{T}=0$ and on outflows we should have $\frac{\partial u_{n}}{\partial n}=$ $\frac{\partial u_{T}}{\partial n}=0$, where the subscripts $n$ and $T$ denote normal and tangential directions to the inflow/outflow, respectively. When calculating the velocity field and the nonNewtonian extra stress tensor, the values of the non-Newtonian extra stress tensor on the boundaries of the domain are required. They are obtained by following the ideas of Tomé et al. [6] as follows. On inflows we assume that $S^{x x}=S^{x y}=S^{y y}=0$ and on outflows we impose $\frac{\partial S^{x x}}{\partial n}=\frac{\partial S^{x y}}{\partial n}=\frac{\partial S^{y y}}{\partial n}=0$. On rigid boundaries, the components of the extra stress tensor are calculated from (2.8)-(2.10) which we assume to hold with the initial condition $\mathbf{S}=\mathbf{0}$. Following Tomé et al. [6], it can be shown that on rigid boundaries parallel to the $x$-axis, $S^{x x}, S^{y y}$ and $S^{x y}$ given by

$$
\begin{aligned}
& S^{y y}(x, y, t+\delta t)=0, \\
& S^{x y}(x, y, t+\delta t)=e^{-\frac{1}{W e} \delta t} S^{x y}(x, y, t)+\frac{1}{2}\left(\frac{\partial u(x, y, t)}{\partial y}-\frac{\partial u(x, y, t+\delta t)}{\partial y}\right)\left[1+e^{-\frac{1}{W e} \delta t}\right], \\
& S^{x x}(x, y, t+\delta t)=e^{-\frac{1}{W e} \delta t} S^{x x}(x, y, t)+\delta t\left[\frac{\partial u(x, y, t)}{\partial y} e^{-\frac{1}{W e} \delta t} S^{x y}(x, y, t)\right. \\
& \left.+\frac{\partial u(x, y, t+\delta t)}{\partial y} S^{x y}(x, y, t+\delta t)\right]+\frac{1}{2} W e\left(\frac{\partial u(x, y, t)}{\partial y}+\frac{\partial u(x, y, t+\delta t)}{\partial y}\right)^{2}\left[1-e^{-\frac{1}{W e} \delta t}\right] .
\end{aligned}
$$


If the rigid boundary is parallel to the $y$-axis the calculation of $S^{x x}, S^{x y}, S^{y y}$ on the rigid boundary is similar to the case above.

\subsection{Free Surface Stress Conditions}

We consider transient free surface flows of viscous fluid flowing into a passive atmosphere and neglect surface tension effects. In this case, the appropriate boundary conditions on the free surface can be written as (see Tomé et al. [6])

$$
\begin{aligned}
& p=\frac{1}{R e}\left\{S^{x x} n_{x}^{2}+2 S^{x y} n_{x} n_{y}+S^{y y} n_{y}^{2}+2\left[\frac{\partial u}{\partial x} n_{x}^{2}+\left(\frac{\partial u}{\partial y}+\frac{\partial v}{\partial x}\right) n_{x} n_{y}+\frac{\partial v}{\partial y} n_{y}^{2}\right]\right\} \\
& \frac{1}{R e}\left\{\left[S^{x x}-S^{y y}+2\left(\frac{\partial u}{\partial x}-\frac{\partial v}{\partial y}\right)\right] n_{x} n_{y}+\left(S^{x y}+\frac{\partial u}{\partial y}+\frac{\partial v}{\partial x}\right)\left(n_{y}^{2}-n_{x}^{2}\right)\right\}=0
\end{aligned}
$$

where $\mathbf{n}=\left(n_{x}, n_{y}\right)$ is the normal outward unit vector to the free surface.

\section{Numerical Method}

To solve equations (2.7)-(2.12) we employ the procedure used by Tomé et al. [6]. We suppose at time $t_{0}$ the velocity field $\mathbf{u}\left(\mathbf{x}, t_{0}\right)$ and the non-Newtonian tensor $\mathbf{S}\left(\mathbf{x}, t_{0}\right)$ are known and the values of $\mathbf{u}$ and $\mathbf{S}$ on the boundary are given. To compute the velocity field $\mathbf{u}(\mathbf{x}, t)$ and the non-Newtonian tensor $\mathbf{S}(\mathbf{x}, t)$, where $t=t_{0}+\delta t$, we proceed as follows:

Step 1: Calculate a tentative velocity field, $\tilde{\mathbf{u}}(\mathbf{x}, t)$, from

$$
\frac{\partial \tilde{\mathbf{u}}}{\partial t}=-\nabla \cdot(\mathbf{u u})-\nabla \tilde{p}+\frac{1}{R e} \nabla^{2} \mathbf{u}+\frac{1}{R e} \nabla \cdot \mathbf{S}+\frac{1}{F r^{2}} \mathbf{g}
$$

with $\tilde{\mathbf{u}}\left(\mathbf{x}, t_{0}\right)=\mathbf{u}\left(\mathbf{x}, t_{0}\right)$ using the correct boundary conditions for $\mathbf{u}\left(\mathbf{x}, t_{0}\right)$. The pressure field $\tilde{p}\left(\mathbf{x}, t_{0}\right)$ can be arbitrary with the restriction that $\tilde{p}\left(\mathbf{x}, t_{0}\right)$ must satisfy the pressure condition on the free surface (see equation (3.1)).

Step 2: Solve the Poisson: $\nabla^{2} \psi(\mathbf{x}, t)=\nabla \cdot \tilde{\mathbf{u}}(\mathbf{x}, t)$. The appropriate boundary conditions for this equation are: $\frac{\partial \psi(\mathbf{x}, t)}{\partial n}=0$ on rigid boundary and inflows and $\psi=0$ on the free surface and outflows.

Step 3: Compute the final velocity: $\mathbf{u}(\mathbf{x}, t)=\tilde{\mathbf{u}}(\mathbf{x}, t)-\nabla \psi(\mathbf{x}, t)$.

Step 4: Compute the pressure: $p(\mathbf{x}, t)=\tilde{p}\left(\mathbf{x}, t_{0}\right)+\frac{\psi(\mathbf{x}, t)}{\delta t}$.

Step 5: Update the components of the non-Newtonian extra stress tensor according to the equations derived in Section 3.1.

Step 6: Compute the components of the extra-stress tensor using equations (2.8)(2.10).

Step 7: Update the marker particles positions. The last step in the calculation is to move the marker particles to their new positions. This is performed by solving $\frac{d x}{d t}=u, \frac{d y}{d t}=v$ by Euler's method. The fluid surface is defined by a list containing these particles and the visualization of the free surface is obtained by connecting them by straight lines. 


\section{Finite Difference Approximation}

The equations describing the numerical method presented in Section 4 will be solved by the finite difference method on a staggered grid (see Figure 1a.). The velocity $u$ and $v$ are staggered by $\delta x / 2$ and $\delta y / 2$ while the pressure and the components of the non-Newtonian extra stress are located at cell centres. A scheme for identifying the fluid region and the free surface is employed. To effect this the cells in the mesh can be of several types, namely: cells Full of fluid (F), Surface cells (S), Empty cells (E), Inflow cells (I), Outflow cells (O) and Boundary cells (B). The F-cell is required to contain fluid and to have no E-cell face in contact with any of its faces and S-cells are defined to contain fluid and to have at least one face in contact with an E-cell face. I-cells define an inflow boundary while O-cells define an outflow boundary. B-cells define a rigid boundary where the no-slip condition is imposed. Figure 1b displays the types of cells within the mesh.

a)

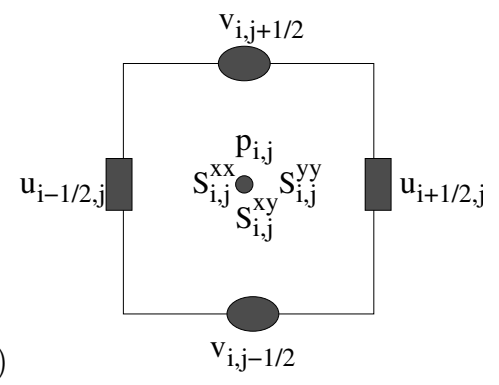

b)

\begin{tabular}{|c|c|c|c|c|c|c|c|c|c|c|c|c|}
\hline & & & & & & & $\begin{array}{ll}\mathrm{B} \\
\end{array}$ & & \multicolumn{2}{|c|}{\begin{tabular}{|l|l|} 
B & B \\
\end{tabular}} & \\
\hline & & & & & B & & $\mathrm{E}$ & $E$ & \begin{tabular}{|l|l}
$E$ \\
\end{tabular} & $E$ & & $\mathrm{EO}$ \\
\hline & & & & & B & & $\mathrm{E}$ & & $E$ & $\mathrm{E}$ & & $\mathrm{EO}$ \\
\hline & B & B & $B$ & $B$ & B & s & s. & S & $\begin{array}{lll}E & E\end{array}$ & $E$ & & $\mathrm{EO}$ \\
\hline 1 & $\mathrm{~F}$ & $\mathrm{~F}$ & $\mathrm{~F}$ & $\mathrm{~F}$ & $\mathrm{~F}$ & & $\mathrm{~F}$ & $F \mid$ & $\begin{array}{lll}S & E \\
\end{array}$ & $E$ & & $\mathrm{EO}$ \\
\hline I & $\mathrm{F}$ & $\mathrm{F}$ & $\mathrm{F}$ & $\mathrm{F}$ & $\mathbf{F}$ & & $\mathrm{F} F$ & $F$ & $\$ E$ & $E$ & & $\mathrm{EO}$ \\
\hline I & $\mathrm{F}$ & $\mathrm{F}$ & $\mathrm{F}$ & $\mathrm{F}$ & $F$ & & F & 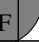 & $\begin{array}{lll}S & E \\
\end{array}$ & $E$ & & $\mathrm{EO}$ \\
\hline & B & B & B & $B$ & B & & $S$ & & $\begin{array}{lll}E \\
\end{array}$ & $\mathrm{E}$ & & $\mathrm{EO}$ \\
\hline & & & & & B & & $\mathrm{E} \mid$ & & $\begin{array}{ll}E \\
\end{array}$ & \begin{tabular}{|l|l}
$E$ & $E$
\end{tabular} & & $\mathrm{EO}$ \\
\hline & & & & & D & & $\mathrm{E}$ & & $\begin{array}{lll}E \\
\end{array}$ & & & $\mathrm{EO}$ \\
\hline & & & & & & & & & $\begin{array}{ll}3 & \mathrm{~B}\end{array}$ & B & & \\
\hline
\end{tabular}

Figure 1: Cells in the computacional domain.

We point out that when solving the equations (4.1) it is usual to approximate the time derivative by the explicit Euler method (see Tomé et al. [6]). In this work we shall approximate the time derivative in (4.1) by the explicit modified Euler method which is second order accurate. The pressure gradient and the linear terms of the momentum equations are approximated by central differences. For the convective terms we employ the high-order upwind VONOS (Variable-Order Non-Oscillatory Scheme) method ([7]). The terms involving the divergent of the non-Newtonian extra stress are approximated by central differences, namely,

$$
\left.\frac{\partial S^{x x}}{\partial x}\right|_{i+\frac{1}{2}, j}=\frac{S_{i+1, j}^{x x}-S_{i, j}^{x x}}{\delta x},\left.\quad \frac{\partial S^{x y}}{\partial y}\right|_{i+\frac{1}{2}, j}=\frac{S_{i+\frac{1}{2}, j+\frac{1}{2}}^{x y}-S_{i+\frac{1}{2}, j-\frac{1}{2}}^{x y}}{\delta y}
$$

where terms like $S_{i+\frac{1}{2}, j+\frac{1}{2}}^{x y}$ are obtained by averaging the four nearest values, e.g. $S_{i+\frac{1}{2}, j+\frac{1}{2}}^{x y}:=\left(S_{i, j}^{x y}+S_{i+1, j}^{x y}+S_{i, j+1}^{x y}+S_{i+1, j+1}^{x y}\right) / 4$. However, if the cell $(i, j)$ is adjacent to a B-Cell or to an E-cell, a forward difference or a backward difference is used to approximate the derivatives $\left.\frac{\partial S^{x y}}{\partial y}\right|_{i+\frac{1}{2}, j}$ and $\left.\frac{\partial S^{x y}}{\partial x}\right|_{i, j+\frac{1}{2}}$. Therefore, the 
$u$-momemtum equation is approximated by

$$
\begin{gathered}
u_{i+\frac{1}{2}, j}^{*}=u_{i+\frac{1}{2}, j}+\delta t F(u, v), \quad \tilde{u}_{i+\frac{1}{2}, j}=u_{i+\frac{1}{2}, j}+\frac{\delta t}{2}\left[F\left(u^{*}, v^{*}\right)+F(u, v)\right], \\
F(u, v)=\left[-\left(\operatorname{conv}\left(u^{2}\right)+\operatorname{conv}(u v)\right)_{i+\frac{1}{2}, j}-\frac{\tilde{p}_{i+1, j}-\tilde{p}_{i, j}}{\delta x}+\left.\frac{1}{R e}\left(\frac{\partial S^{x x}}{\partial x}+\frac{\partial S^{x y}}{\partial y}\right)\right|_{i+\frac{1}{2}, j}\right. \\
\left.+\frac{1}{R e}\left(\frac{u_{i-\frac{1}{2}, j}-2 u_{i+\frac{1}{2}, j}+u_{i+\frac{3}{2}, j}}{\delta x^{2}}+\frac{u_{i+\frac{1}{2}, j-1}-2 u_{i+\frac{1}{2}, j}+u_{i+\frac{1}{2}, j+1}}{\delta y^{2}}\right)+\frac{1}{F r^{2}} g_{x}\right] .
\end{gathered}
$$

The $v$-momentum equations is approximated in the same manner. We would like to point out that the approximations described for discretizing the momentum equations are second order accurate in time and space. In a similar manner, the components of the non-Newtonian extra stress eqs. (2.8)- (2.10) are approximated by finite differences. The time derivative is explicitly approximated by the modified Euler method, the convective terms are computed using the VONOS method [7] and the spatial first order derivatives are second order approximated. For instance, the component $S^{x x}$ is computed as follows:

$$
\begin{aligned}
S_{i, j}^{x x^{*}} & =S_{i, j}^{x x}+\delta t H_{1}\left(S^{x x}, S^{x y}, S^{y y}\right), \\
S_{i, j}^{x x^{(n+1)}} & =S_{i, j}^{x x}+\frac{\delta t}{2}\left[H_{1}\left(S^{x x}, S^{x y}, S^{y y}\right)+H_{1}\left(S^{x x^{*}}, S^{x y^{*}}, S^{y y^{*}}\right)\right] .
\end{aligned}
$$

We observe that the time derivative on the right hand side of equations (2.8)- (2.10) is going to be multiplied by $\delta t$ and we suppose its contribution will be small and therefore it is neglected. Thus, the function $H_{1}\left(S^{x x}, S^{x y}, S^{y y}\right)$ can be written as

$$
\begin{aligned}
& H_{1}\left(S^{x x}, S^{x y}, S^{y y}\right)=-\left(\frac{1}{W e}\right) S_{i, j}^{x x}-\left[\operatorname{conv}\left(u S^{x x}\right)_{i, j}+\operatorname{conv}\left(v S^{x x}\right)_{i, j}-2 \mathrm{D}_{i, j}^{x x} S_{i, j}^{x x}\right. \\
& \left.-2 \frac{\left(u_{i, j+\frac{1}{2}}-u_{i, j-\frac{1}{2}}\right)}{\delta y} S_{i, j}^{x y}\right]-2\left[\operatorname{conv}\left(u \mathrm{D}^{x x}\right)_{i, j}+\operatorname{conv}\left(v \mathrm{D}^{x x}\right)_{i, j}-2\left(\mathrm{D}^{x x}\right)_{i, j}^{2}\right. \\
& \left.-\left(\frac{u_{i, j+\frac{1}{2}}-u_{i, j-\frac{1}{2}}}{\delta y}-\right)^{2}-\frac{u_{i, j+\frac{1}{2}}-u_{i, j-\frac{1}{2}}}{\delta y} \frac{v_{i+\frac{1}{2}, j}-v_{i-\frac{1}{2}, j}}{\delta x}\right] .
\end{aligned}
$$

In equation (5.3), terms which are not defined at cell position are obtained by averaging, e.g.

$$
u_{i, j+\frac{1}{2}}:=\frac{u_{i+\frac{1}{2}, j}+u_{i+\frac{1}{2}, j+1}+u_{i-\frac{1}{2}, j}+u_{i-\frac{1}{2}, j+1}}{4} .
$$

Equations (2.9) and (2.10) are approximated in a similar manner. The Poisson equation (see Step 2 Section 4) is discretized at cell centres using the five-point Laplacian, namely,

$$
\frac{\psi_{i+1, j}-2 \psi_{i, j}+\psi_{i-1, j}}{\delta x^{2}}+\frac{\psi_{i, j+1}-2 \psi_{i, j}+\psi_{i, j-1}}{\delta y^{2}}=\frac{\tilde{u}_{i+\frac{1}{2}, j}-\tilde{u}_{i-\frac{1}{2}, j}}{\delta x}+\frac{\tilde{v}_{i, j+\frac{1}{2}}-\tilde{v}_{i, j-\frac{1}{2}}}{\delta y} .
$$

Equation (5.4) leads to a symmetric and positive definite linear system for $\psi_{i, j}$. In order to solve this linear system we employ the conjugate gradient method. The final velocities are given by

$$
u_{i+\frac{1}{2}, j}^{n+1}=\tilde{u}_{i+\frac{1}{2}, j}-\left(\frac{\psi_{i+1, j}-\psi_{i, j}}{\delta x}\right), \quad v_{i, j+\frac{1}{2}}^{n+1}=\tilde{v}_{i, j+\frac{1}{2}}-\left(\frac{\psi_{i, j+1}-\psi_{i, j}}{\delta y}\right)
$$


and the pressure is calculated by $p_{i, j}=\widetilde{p}_{i, j}+\frac{\psi_{i, j}}{\delta t}$. The free surface stress conditions eqs. (3.1) and (3.2) are approximated in the same way as in Tomé et al. [6].

\section{Validation and Numerical Results}

We validate the numerical technique presented in this paper by simulating the flow in a two-dimensional channel governed by the Maxwell constitutive equation. We consider a 2D-channel formed by two parallel walls at a distance $L$ from each other and having a length of $10 \mathrm{~L}$. At the channel entrance we impose the analytical profiles of fully developed flow given by

$$
\begin{aligned}
& u(y)=-4 \frac{U}{L^{2}}\left(y-\frac{L}{2}\right)^{2}+U, \quad v=0, \\
& S^{x x}(y)=2 W e\left(\frac{\partial u}{\partial y}\right)^{2}, \quad S^{x y}=0, \quad S^{y y}=0 .
\end{aligned}
$$

On the channel walls the no-slip condition is imposed and at the channel exit the conditions for outflow boundaries are applied (see Section 3). To simulate this problem the following input data was employed: $L=1, U=1, \nu=\mu_{0} / \rho=2$, $\rho=1, \lambda=0.4$ Hence $R e=L U / \nu=0.5$ and $W e=\lambda U / L=0.4$. To demonstrate the convergence of the numerical method we ran this problem using three meshes as follows: Mesh $1-\delta x=\delta y=0.2(50 \times 5$ cells $) ;$ Mesh2 $-\delta x=\delta y=0.1(100 \times 10$ cells $)$ and Mesh $3-\delta x=\delta y=0.05(200 \times 20$ cells $)$. We started with the channel empty and injected fluid at the inflow until the channel became full and the steady state was reached. Under steady state conditions the velocity field and the viscoelastic extra-stress on the channel must have the same values as those imposed at the inflow. Figure 2 displays the values of the velocity field and the values of the nonNewtonian extra-stress components $S^{x x}$ at the line $x=5$ (middle of the channel) together with the respective analytic values (see eqs. (6.1), (6.2)). The solid lines in Figure 2 are the analytic solutions while the symbolds represent the numerical solutions of the velocity field and the extra-stress component $S^{x x}$ at the position $x=5$ using the three meshes. As we can see in Figure 2 the agreement between the exact and the numerical solutions is very good. Indeed, the relative $l_{2}$-norm of the error

$$
E^{x x}=\frac{\sum\left(S_{\text {exact }}^{x x}-S_{\text {numerical }}^{x x}\right)^{2}}{\sum\left(S_{\text {exact }}^{x x}\right)^{2}}
$$

are displayed in Table I where we can see that the errors decrease with mesh refinement. These results demonstrate the convergence of the numerical method presented in this paper.

\section{Table I}

\begin{tabular}{|c|c|c|c|}
\hline & Mesh1 & Mesh2 & Mesh3 \\
\hline$E\left(S^{x x}\right)$ & $1.166910^{-2}$ & $8.520010^{-4}$ & $5.600010^{-5}$ \\
\hline
\end{tabular}


a)

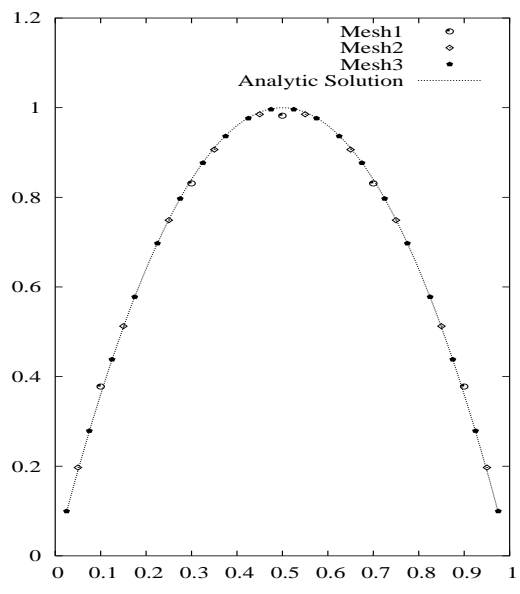

b)

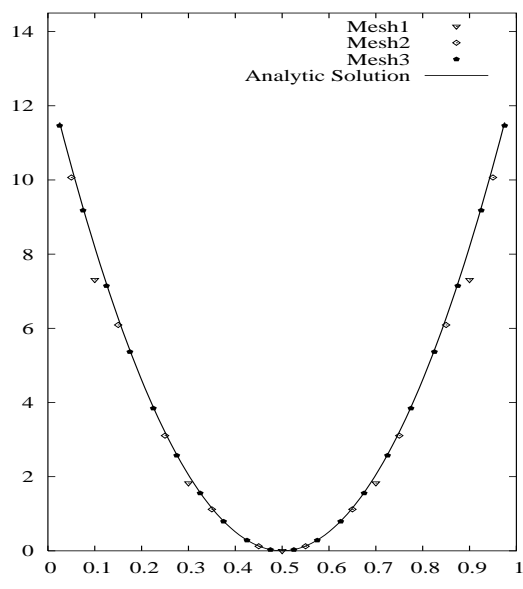

Figure 2: Numerical solution of 2D channel flow: Comparison between numerical and analytic solutions. $u(y)$ velocity (left) and $S^{x x}(y)$ extra-stress component (right).

\subsection{Numerical Simulation of jet buckling}

To demonstrate that the technique presented in this paper can simulate viscoelastic free surface flows we applied it to simulate the buckling instability of thin jets. This problem has been investigated by several authors (e.g. Cruickshank [3], Tomé et al. [6]) and a theory explaining this instability fully has not yet been presented. However, Cruickshank [3] have presented experimental and theoretical estimates predicting when a two-dimensional Newtonian jet will buckle. These estimates were based upon the jet length $(L)$, the height of the inlet to the rigid plate $(H)$ and the Reynolds number. From their study they concluded that if both conditions $R e<0.5$ and $H / D>10$ then a two-dimensional Newtonian jet will buckle. To illustrate that viscoelasticity has a strong influence on the jet buckling phenomenon we present three calculations: one calculation for a Newtonian fluid; the other two calculations we used the Maxwell and the Oldroyd-B constitutive equations. In these calculations we used the following input data: jet length $L=6 \mathrm{~mm}$, inlet velocity $U=0.25 \mathrm{~ms}^{-1}$, height of the inlet to the rigid plate $H=5 \mathrm{~cm}$, mesh spacing $\delta x=\delta y=1 \mathrm{~mm}$. The Newtonian fluid was defined by having viscosity $\mu_{0}=6$ Pas and fluid density $\rho=1000 \mathrm{kgm}^{-3}$ and therefore $\nu=0.006 \mathrm{~m}^{2} \mathrm{~s}^{-1}$. The Maxwell model was defined by having $\lambda=0.006 \mathrm{~s}$ and the Oldroyd-B model by the constants $\lambda_{1}=\lambda$ and $\lambda_{2}=0.1$ and $\mu_{0}$ was used for both the Maxwell and Oldroyd-B fluids. The scaling parameters were $L, U, \lambda$ and $\nu$, giving $R e=U L / \nu=$ $0.25, W e=\lambda U / L=0.25$. In this case, the ratio $H / L=8.3$ which does not satisfy Cruickshank's second condition and therefore the Newtonian jet should not buckle. The results of these calculations are displayed in Figure 3. Indeed, Figure 3 shows that the Newtonian jet does not undergo buckling confirming Cruickshank's prediction. However, the viscoelastic jets modelled by the Maxwell and the Oldroyb- 
B constitutive equations did undergo buckling. Moreover, the Maxwell jet starts buckling by initially bending to the right while the Oldroyd-B jet bends first to left. These results show that the buckling instability when it occurs has no preferencial direction. We believe that these results are due to the viscoelastic effects of the Maxwell and Oldroyd-B models confirming that the numerical method presented in this paper can indeed simulate flows governed by the Maxwell constitutive equation.

Newtonian
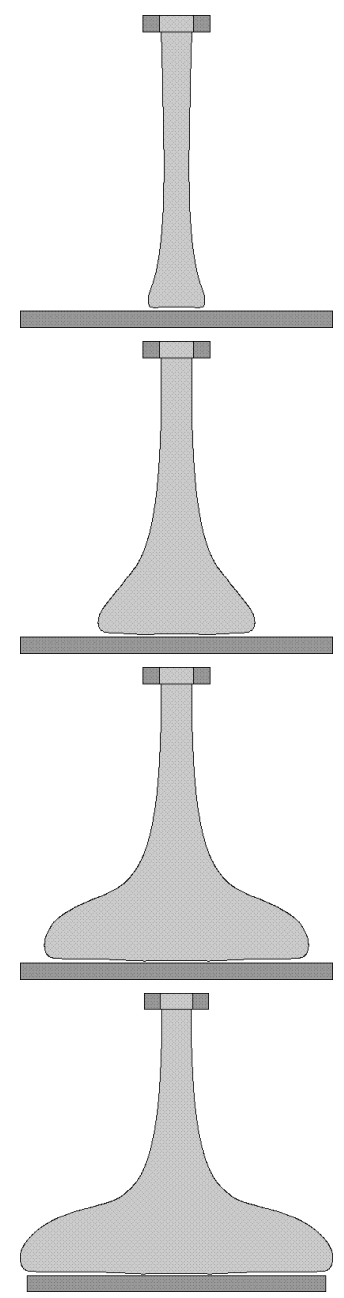

Maxwell
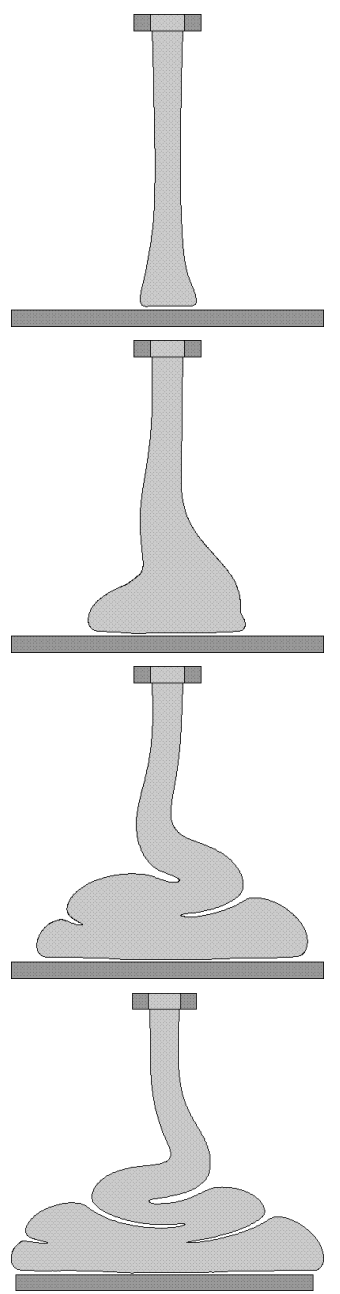

Oldroyd-B

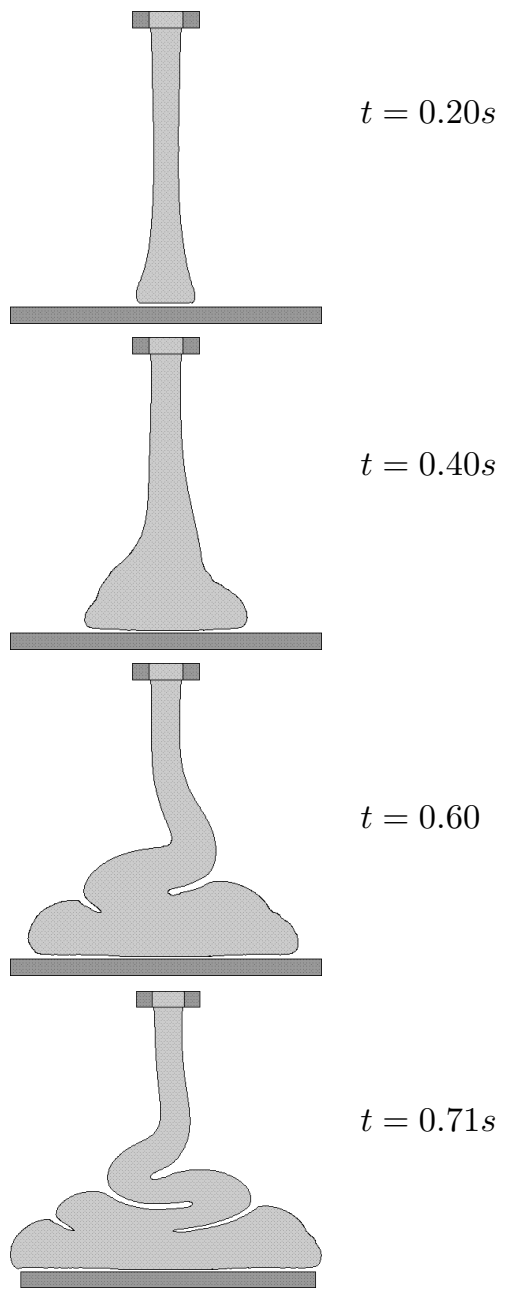

Figure 3: Numerical simulation of jet buckling: Fluid flow configuration at different times. $R e=0.25, \mathrm{We}=0.25$. 


\section{Concluding Remarks}

This paper has been concerned with the development of a numerical method for solving the upper convected Maxwell equation for free surface flows. The finite difference method was validated by simulating the flow of a Maxwell fluid in a $2 \mathrm{D}$ channel. The numerical results were compared with analytic solutions and good agreement was obtained. Mesh refinement was performed which showed the convergence of the method. The problem of jet buckling was simulated and it was found that viscoelasticity has a strong effect on the jet buckling instability.

Resumo. Neste trabalho é apresentado uma técnica numérica utilizando o método de diferenças finitas para simular escoamentos bidimensionais com superfícies livres para um fluido viscoelástico do tipo Maxwell. São apresentados as equações governantes juntamente com as condições de contorno, as formulações para o cálculo do tensor extra-tensão em contornos rígidos e as condições para a superfície livre. Resultados numéricos demonstrando a capacidade desta técnica na simulação de escoamentos viscoelásticos tipo Maxwell são apresentados. Também são apresentados resultados numéricos que mostram a convergência do método numérico.

\section{References}

[1] A.A. Amsden and F.H. Harlow, "The SMAC method: a numerical technique for calculating incompressible fluid flow", Los Alamos Scientific Laboratory, Report LA, 4370, 1970.

[2] M.J. Crochet and R. Keunings, Die swell of a Maxwell fluid - numerical prediction, J. Non-Newtonian Fluid Mech., 7 (1980), 199-212.

[3] J.O. Cruickshank, Low-Reynolds-number instabilities in stagnating jet flows, J. Fluid. Mech., 193 (1988), 111-127.

[4] M. Griebel, T. Dornseifer and T. Neunhoeffer, "Numerical Simulation in Fluid Dynamics: a practical introduction", SIAM publications, 1997.

[5] G. Mompean and M. Deville, Unsteady finite volume of Oldroyd-B fluid through a three-dimensional planar contraction, J. Non-Newtonian Fluid Mech., 72 (1997), 253-279.

[6] M.F. Tomé, N. Mangiavacchi, J.A. Cuminato, A. Castelo and S. Mckee, A finite difference technique for simulating unsteady viscoelastic free surface flows, $J$. Non-Newtonian Fluid Mech, 106 (2002), 61-106.

[7] A. Varonos and G. Bergeles, Development and assessment of a variable-order non-oscillatory scheme for convection term discretization, Intern. J. Numer. Meth. Fluids, 26 (1998), 1-16.

[8] J.Y. Yoo and Y. Na, A numerical study of the planar contraction flow of a viscoelastic fluid using the SIMPLER algorithm, J. Non-Newtonian Fluid Mech., 30 (1991), 89-106. 